\title{
The proper motion of the isolated neutron star RX J1605.3+3249 (Research Note)
}

\author{
S. Zane ${ }^{1}$, A. De Luca ${ }^{2}$, R. P. Mignani ${ }^{1}$, and R. Turolla ${ }^{3}$ \\ 1 Mullard Space Science Laboratory, Holmbury St. Mary, Dorking - Surrey, RH5 6NT, UK \\ e-mail: [sz;rm]@mssl.ucl.ac.uk \\ 2 Istituto di Astrofisica Spaziale e Fisica Cosmica, via Bassini 15, 20133 Milan, Italy \\ e-mail: deluca@iasf-milano.inaf.it \\ 3 Universitá di Padova, Dipartimento di Fisica, via Marzolo 8, 35131 Padova, Italy \\ e-mail: roberto.turolla@pd.infn.it
}

Received 12 April 2006 / Accepted 29 June 2006

\section{ABSTRACT}

\begin{abstract}
Aims. We present a new optical observation of the X-ray dim isolated neutron star RX J1605.3+3249, performed in 2005 with the Wide Field Channel (WFC) of the Advanced Camera for Surveys (ACS) on board Hubble Space Telescope, aimed at measuring the neutron star proper motion.

Methods. We have compared the target position with the one measured in a previous HST image of the field obtained in 2001.

Results. We infer a proper motion of $\mu=155.0 \pm 3.1 \mathrm{mas} / \mathrm{yr}$, corresponding to a star velocity $V_{\mathrm{T}} \sim 73 \mathrm{~km} \mathrm{~s}^{-1}$ at $100 \mathrm{pc}$ distance. Our measurement refines the result recently reported by Motch et al. (2005, A\&A, 429, 257), and confirms the optical source as the counterpart of the neutron star.
\end{abstract}

Key words. stars: neutron - ultraviolet: stars

\section{Introduction}

Since their discovery, neutron stars (NSs) have been regarded as natural laboratories to foster our understanding of both quantum chromodynamics (QCD) and quantum electrodynamics (QED). In particular, by combining the information on the star mass and radius it is possible to constrain the equation of state (EOS) at supra-nuclear densities, thus test QCD and probe the properties of the superfluid in the star interior. Up to now, reliable determinations on the neutron star mass have been obtained from pulsars in binary systems and are in good agreement with theoretical expectations (see Stairs 2004; Nice et al. 2005). A direct measure of the star radius can be achieved in those neutron stars from which surface thermal emission is detected, provided that the distance is known. For this reason, "truly" isolated neutron stars whose (faint) surface emission is not contaminated by accretion from a binary companion or by the presence of a surrounding supernova remnant are ideal targets for this kind of studies.

In this respect, one of the major outcomes of the ROSAT all sky survey has been the discovery of seven close-by, dim X-ray isolated neutron stars (hereafter XDINSs) which stand apart with respect to other known classes of neutron stars detected at X-ray energies (see Treves et al. 2000; Haberl 2004; Zane et al. 2005). The XDINSs feature: (i) thermal, blackbody-like, spectrum with $k T \sim 100 \mathrm{eV}$; (ii) large $\mathrm{X}$-ray to optical flux ratio, $f_{\mathrm{X}} / f_{V}>10^{3}$; (iii) low X-ray luminosity, $L_{\mathrm{X}} \approx 10^{30}-10^{31} \mathrm{erg} \mathrm{s}^{-1}$; (iv) low column density, $N_{\mathrm{H}} \sim 10^{20} \mathrm{~cm}^{-2}$; (v) no association with a supernova remnant, (vi) no evidence for non-thermal hard X-ray tails,

* Based on observations with the NASA/ESA Hubble Space Telescope, obtained at the Space Telescope Science Institute, which is operated by AURA, Inc. under contract No. NAS 5-26555. a clue that is interpreted as the absence of an active magnetosphere. All of them are radio-silent and, thus far, pulsations have been detected in five sources, with periods in the range 3-11 s.

XDINSs were unanimously believed to be steady sources, as indicated by several years of observations for the brightest of them. Unexpectedly, and for the first ever time, XMM-Newton observations have recently revealed a substantial change in the spectral shape and pulse profile of the second most luminous source, RX J0720.4-3125, over a timescale of $\sim 2 \mathrm{yr}$ (De Vries et al. 2004; Vink et al. 2004, 2005), which are likely caused by the precession of the star with a period of $\sim 7 \mathrm{yr}$ (Haberl et al. 2006). Possible variations in the pulse profile of RX J0420.0-5022 over a similar timescale ( $\sim 0.5 \mathrm{yr})$ have also been reported, although only at a low significance level (Haberl et al. 2004).

Although the X-ray thermal emission of XDINSs is generally recognised to originate from the star surface, its nature is still under debate. Different possibilities have been discussed: i) accretion of the interstellar medium onto old $\left(\gtrsim 10^{7} \mathrm{yr}\right)$ neutron stars, ii) the cooling of much younger $\left(\approx 10^{5} \mathrm{yr}\right)$ objects, or iii) dissipation of a decaying super-strong magnetic field $\left(B \gtrsim 10^{13} \mathrm{G}\right)$. In the latter case, XDINSs may be old magnetars (aka highly magnetized neutron stars, maybe genetically related to Anomalous X-ray Pulsars and Soft $\gamma$-ray Repeaters). Broad absorption features, which strength appears to vary with the pulse phase, have been indeed detected at energies in the range $\sim 200-700 \mathrm{eV}$ in all pulsating XDINSs and are usually interpreted in terms of proton cyclotron resonances or boundbound transitions in light-element atoms. The inferred magnetic field is quite strong, up to $B \sim 10^{14} \mathrm{G}$ (van Kerkwijk et al. 2004; Haberl et al. 2004; Zane et al. 2005), lending support to the 
AXPs-SGRs-XDINSs connection. A (slightly) more direct estimate, based on the spin-down measure, has been obtained so far only in RX J0720.4-3125 and RX J1308.6+2127 (Cropper et al. 2004; Kaplan \& van Kerkwijk 2005a,b). Again, the resulting field strength is $B \sim(2-3) \times 10^{13} \mathrm{G}$, of order of the QED limit $\left(B_{\mathrm{OED}} \simeq 4.4 \times 10^{13} \mathrm{G}\right)$.

In the light of this, XDINSs promise deeper insights into the structure and thermal evolution of NSs. This is why, in recent years, much effort has been devoted to extend their multiwavelength coverage, especially in the optical by exploiting new-generation telescopes. Optical identifications open the possibility of a direct measure of the source distance, through parallax, and in turn of the star radius. A distance measurement based on the optical parallax has been already obtained for the brightest source, the prototype of the class RX J1856.5-3754 (Walter 2001; Walter \& Lattimer 2002). This led to a result of the uttermost importance, i.e., the direct estimate of the star velocity and radius. Similar measurements for the second brightest source, RX J0720-3125, are currently under way. Its proper motion has been recently obtained from ground-based observations, yielding a stringent lower limit on the star velocity and a possible birth location (Motch et al. 2003).

RX J1605.3+3249 is, together with RX J1308.6+2127, one of the latest additions to the list of XDINSs for which an optical counterpart has been proposed. Preliminary HST observations revealed a rather blue object at the position of RX J1605.3+3249 (Kaplan et al. 2003), and the optical points lay above the Rayleigh-Jeans tail of the blackbody which fits the X-ray spectrum. This is similar to what has been found in case of other XDINSs, e.g. for RX J1856.5-3754 (van Kerkwijk \& Kulkarni 2001) and RX J0720.4-3125 (Motch et al. 2003), making the association likely but certainly not yet compelling. A more certain identification came last year, when Motch et al. (2005) measured a proper motion of $\mu=144.5 \pm 13.2 \mathrm{mas} / \mathrm{yr}$, based on deep optical imaging obtained with the Subaru telescope in 1999 and 2003.

Here we present new observations of RX J1605.3+3249, obtained during 2005 with the Wide Field Channel of the Advanced Camera for Surveys on board Hubble Space Telescope. Using the HST image first obtained in 2001 by Kaplan et al. (2003) we derive an accurate measure of the star proper motion, which provides further evidence that this is an isolated neutron star.

\section{The proper motion measure}

\subsection{Observations and data analysis}

Our HST observations of the field of RX J1605.3+3249 were performed on 2005, February 6 in two consecutive telescope orbits. In each visit the integration time was of $\sim 2400 \mathrm{~s}$, split in four exposures of $\sim 600 \mathrm{~s}$ each to allow for cosmic ray cleaning. The target was imaged with the Wide Field Channel (WFC) of the Advanced Camera for Surveys (ACS) instrument (Gonzaga et al. 2005), a two-CCD detector offering a combined field of view of $200^{\prime \prime} \times 200^{\prime \prime}$ with an average pixel size of $\sim 50$ mas. The broad-band $V$ filter $F 606 W\left(\lambda_{0}=5907 \mathrm{~A}, \Delta \lambda=2342 \mathrm{~A}\right)$ was used to match the spectral band where the optical counterpart was detected. The ACS data were retrieved from the STScI archive after default pipeline reduction (de-biassing, flatfielding) and photometric calibration. The STSDAS drizzle task was used to combine single exposures and to produce a mosaic image from the two ACS chips after correcting for the geometric distortion of the detector (Gonzaga et al. 2005). The same procedure allowed to reject cosmic ray hits. Further

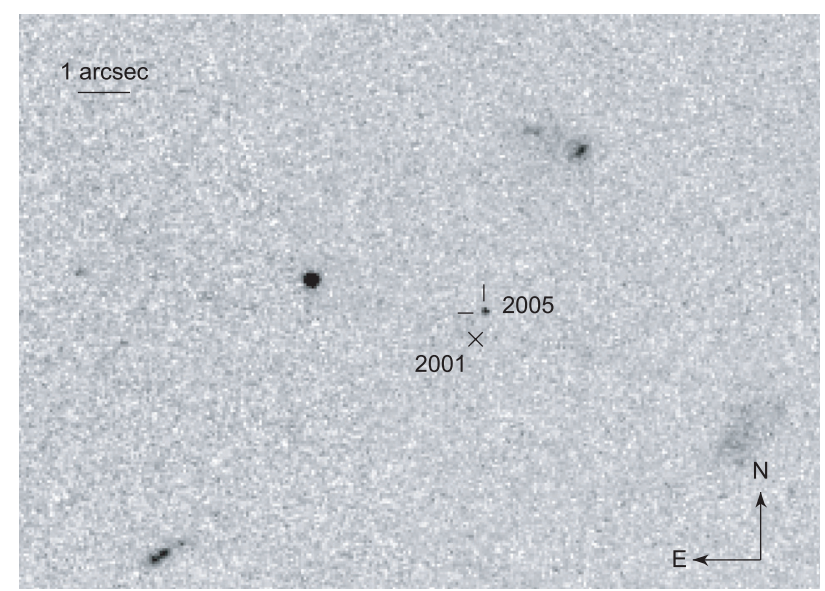

Fig. 1. HST/ACS image of the field of RX J1605.3+3249 taken with the F606W filter and a total integration time of $4800 \mathrm{~s}$ in 2005 (North to the top, East to the left). The position of the target as measured in 2001 and 2005 is shown.

cleaning from residual cosmic ray traces was performed off-line using specific algorithms in IRAF. Our target is clearly detected in the final ACS image (see Fig. 1).

The first-epoch dataset to be used for the proper motion measurement is provided by the HST/STIS observations of Kaplan et al. (2003), performed on 2001, July 21 using the CCD detector $\left(52^{\prime \prime} \times 52^{\prime \prime}\right.$ FOV, $\sim 50$ mas/pixel $)$. These observations were performed with the unfiltered 50CCD and with the longpass $F 28 X 50 L P$ filters (transmitting photons at $\lambda>5500 \mathrm{~A}$ ), with total integration times of $2700 \mathrm{~s}$ (split in 4 exposures) and $5400 \mathrm{~s}$ (split in 8 exposures), respectively. We decided to use the unfiltered STIS observations since the target is detected with a higher signal-to-noise (Kaplan et al. 2003). The STIS data were retrieved from the STScI Public Archive after on-theflight re-calibration using the most appropriate reference files, which included a correction for the CCD geometric distortion (Kim Quijano et al. 2003). Individual, co-aligned images (about $670 \mathrm{~s}$ integration time) were then registered and combined using a median filter to reject cosmic ray hits. As for the ACS data, residual hits in the resulting image were removed using specific cosmic-ray subtraction algorithms in IRAF. The final STIS image was then taken as the reference for the proper motion measurement and was aligned in Right Ascension (RA) and Declination (Dec) according to the HST roll angle information.

\subsection{The relative astrometry}

In order to perform an accurate image superposition, we followed the same approach we successfully applied in several previous astrometric studies of several neutron stars (e.g., Caraveo et al. 1996; De Luca et al. 2000; Caraveo et al. 2001; Caraveo \& Mignani 1999, for the proper motion and parallax of Geminga and Vela, and for the proper motion of the Crab).

As a first step, we defined a relative reference grid using the pixel coordinates of a set of common, appropriate reference sources selected on the images. Such reference objects need to be point-like, not saturated and, in order not to introduce systematics in the frame registration, uniformly distributed across the field. We identified 18 such sources in common between the STIS and ACS images, this number being constrained by the smaller STIS field of view. For all of them, we computed their positions by fitting their intensity profile with a 2D Gaussian function using optimized areas. The centering accuracy of the 
reference sources was of the order of 0.03-0.10 pixel (per coordinate) in both the STIS and ACS images, depending on the object signal-to-noise. The coordinates of the optical counterpart to RX J1605.3+3249 were computed in the same way with accuracies of the order of $0.03-0.08$ pixels per coordinate. In the next step, the ACS-to-STIS frame registration was performed computing a linear coordinate transformation between the two reference grids, fitting 5 parameters (namely, 2 independent shift factors, 2 independent scale factors and a rotation angle). The superposition turned out to be very accurate, with rms residuals of 0.088 STIS/CCD pixels and 0.153 STIS/CCD pixels in the $x$ (RA) and $y$ (Dec) directions, respectively. In order to check for systematics, we divided the reference sources into two subsets containing 9 objects each (randomly distributed across the field) and we repeated the frame registration, obtaining statistically undistinguishable results. Moreover, we did not find evidence for peculiar motions for any of the reference sources, nor did we identify peculiar correlations in the reference sources' position residuals. We are thus confident that our frame registration is correct and free of systematics. We note that the accuracy on the reference grid superposition directly measures the remarkable accuracy of the geometric distortion correction applied to the two instruments' fields of view.

\subsection{Results}

After registering the ACS and STIS images to a unique reference frame, we measured the displacement of the optical counterpart of RX J1605.3+3249 by computing the difference in its relative coordinates. The overall uncertainty on such difference was evaluated adding in quadrature the uncertainties of all the astrometric steps quoted in the previous section. These include the centering errors of the target in the STIS image $(0.06$ and 0.08 pixels in RA and Dec, respectively) and in the ACS image (0.05 and 0.03 pixels in RA and Dec, respectively), as well as the frame superposition error (0.09 and 0.16 STIS pixels in RA and Dec, respectively). The overall displacement of the target is thus $-3.05 \pm 0.12$ STIS pixels in RA and 10.40 \pm 0.18 STIS pixels in Dec along the $\sim 3.55$ years time interval between the STIS observations (epoch 2001.553) and the ACS observations (epoch 2005.099). Using the calibrated STIS/CCD plate scale of $0.05073 /$ pixel, with an uncertainty of $0.15 \%$, such a displacement translates to a proper motion $\mu_{\alpha} \cos (\delta)=-43.7 \pm$ $1.7 \mathrm{mas} /$ year, $\mu_{\delta}=148.7 \pm 2.6$ mas/year, corresponding to a total proper motion of $155.0 \pm 3.1 \mathrm{mas} /$ year along a position angle of $\sim 344^{\circ} \pm 1^{\circ}$ (Eastward from North). We have compared our proper motion with the one obtained by Motch et al. (2005) using Subaru observations coupled with the HST/STIS observations also used in this work. Motch et al. (2005) obtained a proper motion of of $144.5 \pm 13.2$ mas/year with a position angle of $\sim 350.14^{\circ} \pm 5.65^{\circ}$. Although these values are somewhat different from ours, they are certainly compatible (within $1 \sigma$ ) with the quoted errors.

\section{Conclusions}

For intrinsically faint objects such as XDINSs, a firm confirmation of the counterpart based on colors is problematic. Indeed, reliable measurements of the optical flux distribution have been obtained so far only for the two brightest sources RX J1856.5-3754 and RX J0720-3125. Thus, alternative approaches should be sought. Searching for optical pulsations at the X-ray period, although ideally the best way, turns out to be extremely difficult owing to the objects' faintness. However, another distinguishing feature of Galactic neutron stars is their high space velocities, with values ranging between $\approx 100$ and $\approx 1000 \mathrm{~km} \mathrm{~s}^{-1}$ (e.g., Arzoumanian et al. 2002), which can imply significant proper motions. The use of proper motion measurements, successfully experimented in the case of the optical identification of Geminga (Bignami et al. 1993), historically proved to be a very powerful tool. Indeed, optical identifications of neutron stars based on proper motion measurements have been obtained for the ordinary pulsars PSR B0656+14 (Mignani et al. 2000) and PSR B1929+10 (Mignani et al. 2002) and for the XDINSs RXJ 1856.5-3754 (Walter 2001) and RX J0720-3125 (Motch et al. 2003). Thus, proper motion measurements, although requiring much longer time spans, are as efficient as timing in securing the optical identification of isolated neutron stars (see, Mignani et al. 2004, for a review).

The measure of the proper motion presented here for RX J1605.3+3249 confirms, and also updates, the original result of Motch et al. (2005). This led to an estimated of the star velocity of $V_{\mathrm{T}} \sim 73(\mathrm{~d} / 100 \mathrm{pc}) \mathrm{km} \mathrm{s}^{-1}$, comparable to that measured for others XDINSs, and in turn to a proper identification of the optical counterpart. Besides, most of the conclusions inferred by Motch et al. (2005) are further strengthened, including the intriguing identification of the Sco OB2 association as the likely birth place for this source and several other XDINSs. Sco OB2 is a rich $\mathrm{OB}$ association, the closest to the Earth, and it is part of the young star complex known as the Gould Belt. If, as proposed, at least three of the XDINSs originated in such region, its presence in the proximity of the Earth may explain the relative overabundance of faint isolated neutron stars detected in the solar proximity.

Finally, we note that Motch et al. (2005) claim the presence of a non-thermal component in the optical spectrum of RX J1605.3+3249, with a power law index of $\sim 1.5$. However, we caution that a firm conclusion cannot be reached yet. In fact, Motch et al. (2005) based their results on the two Subaru points only, while Kaplan et al. (2003) found that the two Hubble Space Telescope points were consistent with a Rayleigh-Jeans distribution. While using all the presently available (HST and Subaru) observations, we find that no acceptable linear fit can be found: our best fit gives a slope of $0.5 \pm 0.5$ at $1 \sigma$, but it has $\chi^{2} \sim 13$ (see Fig. 2) so it is statistically unacceptable. If we exclude the Wide Field Channel HST point we find a slope of $0.6 \pm 0.5$ and $\chi^{2} \sim 5$, still unsatisfactory. We caveat that fluxes have been derived using the zeropoints resulting from standard $H S T$ pipeline photometric calibration. Since the used HST filters are so wide, a more accurate conversion of instrumental magnitudes into fluxes could be computed taking into account the filter bandpass shape (including detector throughput), as well as the source spectral shape (including the effects of interstellar reddening), following e.g. Kaplan et al. (2003). However, since the spectral range covered by the HST and Subaru data is rather narrow, the use of such a more sophisticated approach (which would be, in any case, limited by systematics in the cross-calibration between different instrument and by the a priory unknown source spectral shape), is not likely to allow to better constrain the power law slope.

Thus, the spectral fit is virtually unconstrained by the available measurements. Although there is evidence that the $B-R$ color of RX $\mathrm{J} 1605.3+3249$ is too high to be compatible with a Rayleigh-Jeans distribution (Motch et al. 2005), in order to firmly constraint the slope of optical spectrum further data are needed. A more precise spectral characterization would be 


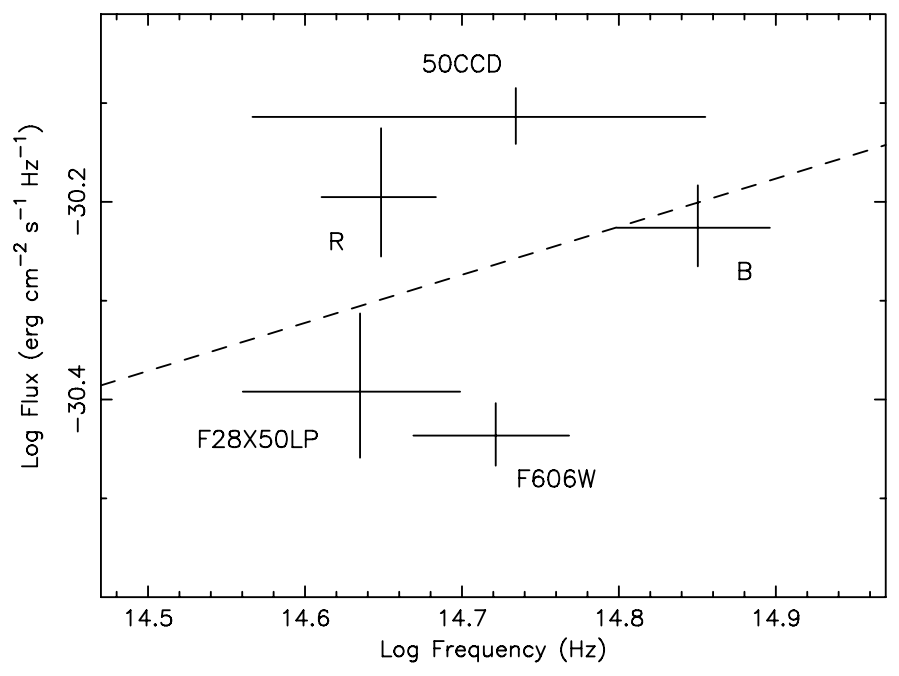

Fig. 2. A collection of the currently available (HST and Subaru) optical observations of RX J1605.3+3249. The dotted line is the best fit power law of slope $0.5 \pm 0.5$. However, this gives a $\chi^{2} \sim 13$ so it is statistically unacceptable (see text for details). A reddening correction with $A_{V}=0.11$ has been applied. Fluxes and central wavelenghts of the large bands of the HST filters have been obtained using the standard photometric calibration, and no systematic errors have been added.

possible only through observations taken with the same telescope and instrument set-up.

Acknowledgements. We are grateful to STScI for allowing us to carry over our programme with ACS after the STIS failure in August 2004. Silvia Zane acknowledges support through a PPARC advanced fellowship.

\section{References}

Arzoumanian, Z., Chernoff, D. F., \& Cordes, J. M. 2002, ApJ, 568, 289 Bignami, G. F., Caraveo, P. A., \& Mereghetti, S. 1993, Nature, 361, 704 Caraveo, P. A., \& Mignani, R. P. 1999, A\&A, 344, 367

Caraveo, P. A., Bignami, G. F., Mignani, R. P., \& Taff, L. G. 1996, ApJ, 461, L91 Caraveo, P. A., De Luca, A., Mignani, R. P., \& Bignami, G. F. 2001, ApJ, 561, 930

Cropper, M., Haberl, F., Zane, S., \& Zavlin, V. E. 2004, MNRAS, 351, 1099

De Luca, A., Mignani, R. P., \& Caraveo, P. A. 2000a, A\&A, 354, 1011

De Luca, A., Mignani, R. P., \& Caraveo, P. A. 2000b, A decade of HST science, ed. M. Livio, K. Noll, \& M. Stiavelli, 32

De Vries, C. P., Vink, J., Mendez, M., \& Verbunt, F. 2004, A\&A, 415, L31

Gonzaga, S., et al. 2005, ACS Instrument Handbook, Version 6.0 (Baltimore: $\mathrm{STScI})$

Haberl, F. 2004, Adv. Sp. Res., 33, 638

Haberl, F., Motch, C., Zavlin, V. E., et al. 2004, A\&A, 424, 635

Haberl, F., Turolla, R., De Vries, C., et al. 2006, A\&A, 451, L17

Kaplan, D. L., Kulkarni, S. R., \& van Kerkwijk, M. H. 2003, ApJ, 588, L33

Kaplan, D., \& van Kerkwijk, M. H. 2005a, ApJ, 628, L45

Kaplan, D., \& van Kerkwijk, M. H. 2005b, ApJ, 635, L65

Kim Quijano, J., et al. 2003, STIS Instrument Handbook, Version 7.0 (Baltimore: STScI)

Mignani, R. P., De Luca, A., \& Caraveo, P. A. 2000, ApJ, 543, 318

Mignani, R. P., De Luca, A., Caraveo, P. A., \& Becker, W. 2002, ApJ, 580, L147

Mignani, R. P., De Luca, A., \& Caraveo, 2004, Young Neutron Stars and Their Environments, held as part of the IAU General Assembly, 14-17 July, 2003 in Sydney, Australia, ed. F. Camilo, \& B. M. Gaensler (San Francisco, CA: ASP), IAU Symp., 218, 391

Motch, C., Zavlin, V. E., \& Haberl, F. 2003, A\&A, 408, 323

Motch, C., Sekiguchi, K., Haberl, F., et al. 2005, A\&A, 429, 257

Nice, D. J., Splaver, E. M., Stairs, I. H., et al. 2005, ApJ, 634, 1242

Stairs, I. H. 2004, Science, 304, 547

Treves, A., Turolla, R., Zane, S., \& Colpi, M. 2000, PASP, 112, 297

van Kerkwijk, M. H., \& Kulkarni, S. R. 2001, A\&A, 378, 986

van Kerkwijk, Kaplan, D. L., Durant, M., Kulkarni, S. R., \& Paerels, F. 2004, ApJ, 608, 432

Vink, J., de Vries, C. P., Mel'ndez, M., \& Verbunt, F. 2004, ApJ, 609, L75

Vink, J., Haberl, F., De Vries, C., et al. 2005, The Astronomer's Telegram, 650

Walter, F. M. 2001, ApJ, 549, 433

Walter, F. M., \& Lattimer, J. M. 2002, ApJ, 576, L145

Zane, S., Cropper, M., Turolla, R., et al. 2005, ApJ, 627, 397 